\title{
Color Image Segmentation for Multimedia Applications
}

\author{
N. IKONOMAKIS \\ Department of Computer and Electrical Engineering, University of Toronto, Canada; \\ e-mail:minoas@dsp.toronto.edu
}

\section{K. N. PLATANIOTIS}

School of Computer Science, Ryerson Polytechnic University, 350 Victoria Street, Toronto, ON, M5B2K3, Canada; e-mail: kplatani@acs.ryerson.ca

\author{
A. N. VENETSANOPOULOS \\ Department of Computer and Electrical Engineering, University of Toronto, 10 King's College Rd., \\ Toronto, ON, M5S 3G4, Canada; e-mail:anv@dsp.toronto.edu
}

(Received: 20 June 1998; in final form: 20 November 1999)

\begin{abstract}
Image segmentation is crucial for multimedia applications. Multimedia databases utilize segmentation for the storage and indexing of images and video. Image segmentation is used for object tracking in the new MPEG-7 video compression standard. It is also used in video conferencing for compression and coding purposes. These are only some of the multimedia applications in image segmentation. It is usually the first task of any image analysis process, and thus, subsequent tasks rely heavily on the quality of segmentation. The proposed method of color image segmentation is very effective in segmenting a multimedia-type image into regions. Pixels are first classified as either chromatic or achromatic depending on their HSI color values. Next, a seed determination algorithm finds seed pixels that are in the center of regions. These seed pixels are used in the region growing step to grow regions by comparing these seed pixels to neighboring pixels using the cylindrical distance metric. Merging regions that are similar in color is a final means used for segmenting the image into even smaller regions.
\end{abstract}

Key words: color image segmentation, region growing, region merging, cylindrical distance metric.

\section{Introduction}

Image segmentation refers to partitioning an image into different regions that are homogeneous or "similar" in some image characteristics. It is an important facet of human visual perception. Humans use their visual sense to partition their environment into distinct objects to help recognize these objects, classify them, guide their movements, and for almost every other visual task. It is a complex process that includes many interacting components. The analysis of color, shape, motion, and texture of objects is usually involved in this process. For the human visual system, this segmentation process is a spontaneous, natural activity. Unfortunately, it is not easy to create artificial algorithms whose performance is comparable to 
that of the human visual system. As Marr has suggested [26], one of the major obstacles to the successful development of theories on segmentation has been a tendency to underestimate the complexity of the problem because the human performance is mediated by methods that are largely subconscious. Because of this, segmentation of images is weakened by various types of uncertainty making the simplest techniques ineffective.

Segmentation is usually the first task of any image analysis process, and thus, subsequent tasks rely heavily on the quality of segmentation. For this reason, a considerable care is taken to improve the probability of a successful segmentation.

Image segmentation has taken a central place in numerous applications, including, but not limited to, multimedia databases, color image and video transmission over the Internet, digital broadcasting, interactive TV, video-on-demand, computer-based training, distance education, video-conferencing and tele-medicine, and, with the development of the hardware and communications infrastructure, to support visual applications. The field has become a principal area of research, not only in electrical engineering, but also in other academic disciplines, such as computer science, geography, medical imaging, criminal justice, and remote sensing.

Most attention to image segmentation has been focused on grey-scale (or monochrome) images. A common problem in the segmentation of grey-scale images occurs when an image has a background of varying grey level, such as, gradually changing shades, or when regions assume some broad range of grey levels. This problem is inherent since intensity is the only available information from monochrome images. It is known that the human eye can detect only in the neighborhood of one or two dozen intensity levels at any point in a complex image due to brightness adaptation, but can differentiate thousands of color shades and intensities [15].

There are currently a large number of color image segmentation techniques available. They can be categorized into four general groups: pixel-based, edgebased, region-based, and model-based techniques. These techniques are either based on concepts of similarity (edge-based) or on discontinuity (pixel-based and region-based) of pixel values. Model-based techniques, where segmentation is posed as a statistical optimization problem, have become popular in the past decade.

Region-based segmentation algorithms include region growing, merging, and splitting/merging techniques. The focus of this paper will be on region-based segmentation. In particular, we will examine region growing and region merging techniques. Pixels are first classified as either chromatic or achromatic depending on their HSI color values. Next, a seed determination algorithm finds seed pixels that are in the center of regions. These seed pixels are used in the region growing step to grow regions by comparing these seed pixels to neighboring pixels using the cylindrical distance metric. Merging regions that are similar in color is a final means used for segmenting the image into even smaller regions. These region-based segmentation techniques will be examined on multimedia-based color 
images. In particular, the results on video-conferencing type color images will be discussed.

\section{Techniques of Color Image Segmentation}

Because of the uncertainty problems encountered while trying to model the human visual system, there are currently a large number of image segmentation techniques that are available. However, no general methods have been found that perform adequately across a varied set of images. The early attempts at grey-scale image segmentation are based on three techniques: pixel-based, region- based, and edgebased techniques. Even though these techniques were introduced three decades ago, they still find great attention in color image segmentation research today. Model-based segmentation techniques have also become popular in the last decade. The following sections will survey the various techniques of color image segmentation starting with pixel-based, edge-based, region-based, and, finally, model-based techniques.

\subsection{PIXEL-BASED TECHNIQUES}

Pixel-based techniques do not consider the spatial context but only decide solely on the basis of the color features at individual pixels [15, 17, 28-30, 36]. This attribute has its advantages and disadvantages. Simplicity of the algorithms is an advantage to pixel-based techniques while the lack of spatial constraints makes them susceptible to noise in the images. Model-based techniques which utilize spatial interaction models to model images are used to further improve pixel-based techniques.

The simplest technique of pixel-based segmentation is histogram thresholding. It is one of the oldest and most popular techniques for image segmentation. An image composed of distinct regions usually has distinct peaks in the color histogram, each corresponding to one region. Adjacent peaks are likely to be separated by valleys. For example, if the image has a distinct object on the background, the color histogram is likely to be bimodal with a deep valley. In this case, called bilevel thresholding [30], the bottom of the valley is taken as the threshold so that pixels that belong above and below this value on the histogram are grouped into two different regions. For multilevel thresholding, the image is composed of a set of distinct regions. The histogram has one or more deep valleys and the selection of the thresholds becomes a problem of detecting valleys. However, detection of the valleys is not a trivial job.

Clustering is another pixel-based technique that is extensively used for image segmentation $[3,19,24,37,41,43]$. The rationale of the clustering technique, is that, typically, the colors in an image tend to form clusters in the histogram. One for each object in the image. In the clustering-based technique, a histogram is first obtained by the color values of all pixels and the shape of each cluster is found. 
Then, each pixel in the image is assigned to the cluster that is closest to the pixel color. Many different clustering algorithms are in existence today [16, 38]. Among these, the $K$-means and the fuzzy $K$-means algorithms have acquired extensive attention $[19,24,41,43]$.

The pixel-based segmentation techniques surveyed above do not consider spatial constraints which make them susceptible to noise in the images. The resulting segmentation often contains isolated, small regions that are not present in noisefree images. In the past decade, many researchers have included spatial constraints in their pixel-based segmentation techniques using statistical models (Section 2.4).

\subsection{EDGE-BASED TECHNIQUES}

Edge-based techniques $[1,5,8,10,15,21,27,34,35,39]$ focus on the discontinuity of a region in the image. The idea of edge-based segmentation techniques is that edges define boundaries and that regions are contained within these edges. Once the edges of regions are found, then the regions could be segmented. Most edge detection techniques are based on finding maxima in the first derivative of the image function or zero-crossings in the second derivative of the image function. Edge-based segmentation techniques are very sensitive to texture variations and impulsive noise.

In a monochrome image, an edge is defined as an intensity discontinuity. Early approaches to color edge detection comprise of extensions from monochrome edge detection techniques which utilize the Sobel operator for finding the first derivative or the Laplacian or Mexican Hat operators to find the second derivative. These techniques were applied to each of the color components independently and then the results were combined using certain logical operations [21].

One common problem with the approaches mentioned previously is that they fail to take into account the correlation among the color channels, and, as a result, they are not able to extract certain crucial information revealed by color. For example, they tend to miss edges that have the same strength but in the opposite direction in two of the three color components. Consequently, the approach to treat the color image as a vector space has been proposed [1, 5, 8, 10, 34, 35, 39]. A color image can be viewed as a 2-dimensional three-channel vector field which can be characterized by a discrete integer function $\vec{f}(x, y)$. The value of this function at each pixel is defined by a 3-dimensional vector in a given color space. Vector space approaches include vector gradient operators $[5,10]$, second derivative operators $[8,21]$, and the entropy operator [34].

\subsection{REGION-BASED TECHNIQUES}

Region-based techniques $[12,15,18,20,40]$ focus on the continuity of a region in the image. Segmenting an image into regions is directly accomplished through region-based segmentation which makes it one of the most popular techniques used 
today. Unlike the pixel-based techniques, region-based techniques consider both color distribution in the color space and spatial constraints. Standard techniques include region growing and split and merge techniques. Region growing is the process of grouping neighboring pixels into regions. The split and merge technique constitutes iteratively splitting the image into smaller and smaller regions and testing to see if adjacent regions need to be merged into one. The process of merging pixels or regions to produce larger regions is usually governed by a homogeneity criterion, such as, a distance measure linked to color similarity.

Region growing is the process of grouping neighboring pixels or a collection of pixels of similar properties into larger regions. Testing for similarity is usually achieved through a homogeneity criterion. Quite often after an image is segmented into regions using a region growing algorithm, the regions are further merged for improved results. A region growing algorithm typically starts with a number of seed pixels in an image and from these it grows regions by iteratively adding unassigned neighboring pixels that satisfy some homogeneity criterion with the existing region of the seed pixel. That is, an unassigned pixel neighboring a region, that started from a seed pixel, may be assigned to that region if it satisfies some color homogeneity criterion. If the pixel is assigned to the region, the pixel set of the region is updated to include this pixel. Region growing techniques differ in choice of a homogeneity criterion and the choice of seed pixels. Several homogeneity criteria linked to color similarity or spatial similarity can be used. These criteria can be defined from local, regional, or global considerations. The choice of seed pixel can be supervised (user-assisted) or unsupervised (automatic).

As opposed to the region growing technique of segmentation, where a region is grown from a seed pixel, the split-and-merge technique subdivides an image initially into a set of arbitrary, disjoint regions and then merges and/or splits the regions in an attempt to satisfy a color homogeneity criterion between the regions. Horowitz and Pavlidis [18] describe a split and merge algorithm, for grey-scale images, that iteratively works toward satisfying these constraints. It will be described here for color images. The image is subdivided into smaller and smaller quadrant regions so that for each region a color homogeneity criterion holds. That is, if for region $R_{i}$ the homogeneity criterion does not hold, divide the region into four subquadrant regions, and so on. This splitting technique may be represented in the form of a so-called quadtree (that is, a tree in which each node has exactly four descendants). The quadtree data structure is the most common used data structure in split and merge algorithms because of its simplicity and computational efficiency [33]. The root of the tree corresponds to the entire image. Merging of adjacent subquadrant regions is allowed if they satisfy a homogeneity criterion. The procedure may be summarized as follows:

1. Split into four disjointed quadrants any region where a homogeneity criterion does not hold.

2. Merge any adjacent regions that satisfy a homogeneity criterion.

3. Stop when no further merging or splitting is possible. 
Most split-and-merge approaches to image segmentation follow this simple procedure with varying approaches coming from different color homogeneity criteria.

\subsection{MODEL-BASED TECHNIQUES}

Recently, much work has been directed toward stochastic model-based techniques $[2,4,6,7,9,13,22,25,31,32]$. In such techniques, the image regions are modeled as random fields and the segmentation problem is posed as a statistical optimization problem. Compared to previous techniques, the stochastic model-based techniques often provide a more precise characterization of the image regions. In fact, various stochastic models can be used to synthesize color textures that closely resemble natural color textures in real-world natural images [7]. Most of the techniques introduced use spatial interaction models like Markov Random Field (MRF) or Gibbs Random Field (GRF) to model digital images. The reports by Cross and Jain [7], S. Geman and D. Geman [13], Cohen and Cooper [6], Derin and Elliott [9], Lakshmanan and Derin [22], Panjwani and Healey [31], Liu and Yang [25], Pappas [32], and Chang et al. [4] all make use of the Gibbs distributions for characterizing MRF. Model-based techniques tend to be computationally intensive and thus are only used in such cases where complexity is not a problem.

\section{Proposed Region-based Segmentation Technique}

We have developed a region-based color image segmentation scheme for multimedia applications. The proposed scheme utilizes the HSI (hue, saturation, intensity) color space because of its close relation to the human perception of colors. Although color receptors in the human eye (cones) absorb light with the greatest sensitivity in the blue, green, and red part of the color spectrum, the signals from the cones are further processed in the visual system [23]. Because of this perception process, a human can easily recognise basic attributes of color: intensity (lightness, or brightness), hue, and saturation. The hue component represents the impression related to the dominant wavelength of the color stimulus. The saturation corresponds to relative color purity. Colors with no saturation are grey-scale colors. Intensity is the amount of light in a color. Maximum intensity is sensed as pure white, while minimum intensity is sensed as pure black. In contrast, the RGB color space has a high correlation between its components (R-B, R-G, G-B). It is hard to visualize a color based on the R, G, and B components. It is also impossible to evaluate the perceived differences between colors on the basis of distance in the RGB color space. In terms of segmentation, the RGB color space is usually not preferred because it is psychologically non-intuitive and non-uniform.

The proposed region-based scheme utilizes region growing and region merging techniques. The scheme can be split into four general steps:

1. The pixels in the image are classified as chromatic or achromatic by examining their HSI color values. 
2. Seed pixels are found for the chromatic region.

3. The region growing algorithm is employed to segment the image into regions.

4. Regions that are similar in color are merged.

Of the three HSI color components of a pixel, hue has the greatest discrimination power because it is independent of any intensity attribute. Even though hue is the most useful attribute, there are two problems in using this value: hue is meaningless when the intensity is very low or very high; and hue is unstable when the saturation is very low [15]. Because of these attributes, in the proposed scheme, the image is first divided into chromatic and achromatic regions by defining effective ranges of hue, saturation, and intensity values in the image. Since the hue value of a pixel is meaningless when the intensity is very low or very high, the achromatic pixels in the image are defined as the pixels that have low or high intensity values. Since hue is unstable for low saturation values, pixels can also be categorized as achromatic if their saturation value is very low. From the concepts discussed above, the achromatic pixels in the HSI color space are defined as follows:

$$
\text { achromatic pixels: } I>90 \text { or } I<10 \text { or } S<10 \text {, }
$$

where the saturation $S$ and intensity $I$ values are normalized from 0 to 100 . Only the intensity values of the achromatic pixels are considered when segmenting the achromatic pixels into regions. Pixels that are not classified as achromatic are automatically categorized as chromatic pixels. For chromatic pixels all the three color values are considered in the algorithm.

\subsection{SEED DETERMINATION}

The region growing algorithm starts with a set of seed pixels and from these grows regions by appending to each seed pixel those neighboring pixels that satisfy a certain homogeneity criterion, which will be described later. An unsupervised algorithm is used to find the 'best' chromatic seed pixels in the image. These pixels will be the pixels that are in the center of the regions in the image. Usually the pixels in the center of a homogeneous region are the pixels that are dominant in color. The algorithm is used only to determine the seeds of the chromatic regions.

The seed determination algorithm employs variance masks to the image on different levels. Only the hue values of the pixels are considered in this algorithm, because, it is the most significant feature that may be used to detect uniform color regions [14]. All the pixels in the image are first considered as level zero seed pixels. At level one, a $3 \times 3$ non-overlapping mask is applied to the chromatic pixels in the image. The mask determines the variance in hue of nine level-zero pixels. If the variance is less than a certain threshold and the nine level zero pixels in the mask are chromatic pixels, then the center pixel of the mask is considered as a level-one seed pixel. The first level seeds represent $3 \times 3$ pixel regions in the image. In the second level, the non-overlapping mask is applied to the levelone seed pixels in the image. Once again, the mask determines the variance in the 
average hue values of the nine level-one seed pixels. If the variance is less than a certain threshold, the center pixel of the mask is considered as a level-two seed pixel and the eight other level-one seeds are disregarded as seeds. The second level seeds represent regions of $9 \times 9$ pixels. The process is repeated for successive level seed pixels until the seed pixels at the last level represent regions of a size just less than the size of the image. Typically, this is level 5 for an image that is a minimum of $3^{5} \times 3^{5}$ in dimension. Figure 1 shows an example of an image with level 1, 2, and 3 seeds. The algorithm is summarized in the following steps, with $a$ representing the level:

1. All chromatic pixels in the image are set as level 0 seed pixels. Set $a$ to 1 .

2. Shift the level $a$ mask to the next nine pixels (beginning with the corner of the image if just increased $a$ ).

3. If the mask reaches the end of the image, increase $a$ and go to step 2 .

4. If all the seed pixels in the mask are of level $a-1$, continue. If not, go to step 2 .

5. Determine the hue variance of the nine level $a-1$ seed pixels in the $3 \times 3$ mask. The variance is computed by considering, if $a=1$, the hue values of the nine pixels. Otherwise, the average hue values of the level $a-1$ seed pixels are considered.

6. If the variance is less than a threshold $T_{\mathrm{VAR}}$, then the center level $a-1$ seed pixel is changed to a level $a$ seed pixel and the other eight level $a-1$ seed pixels are no longer considered as seeds.

7. Go to step 2.

Although the image is not altered in the algorithm, it can be considered as a crude segmentation of the image.

Since hue is considered as a circular value, the variance and average values of a set of hues cannot be calculated using standard linear equations. To calculate the average and variance of a set of hue values, the sum of the cosine and the sine of the nine pixels must first be determined [11]:

$$
\begin{aligned}
& C=\sum_{k=1}^{9} \cos \left(H_{k}\right), \\
& S=\sum_{k=1}^{9} \sin \left(H_{k}\right),
\end{aligned}
$$

where $H_{k}$ is the hue value of pixel $k$ in the $3 \times 3$ mask. The average hue $A V G H U E$ of the nine pixels is then defined as:

$$
A V G H U E= \begin{cases}\arctan (S / C) & \text { if } S>0 \text { and } C>0, \\ \arctan (S / C)+\pi & \text { if } C<0, \\ \arctan (S / C)+2 \pi & \text { if } S<0 \text { and } C>0 .\end{cases}
$$

The variance VARHUE of the nine pixels is determined as follows:

$$
\text { VARHUE }=(-2 \ln (R))^{1 / 2},
$$




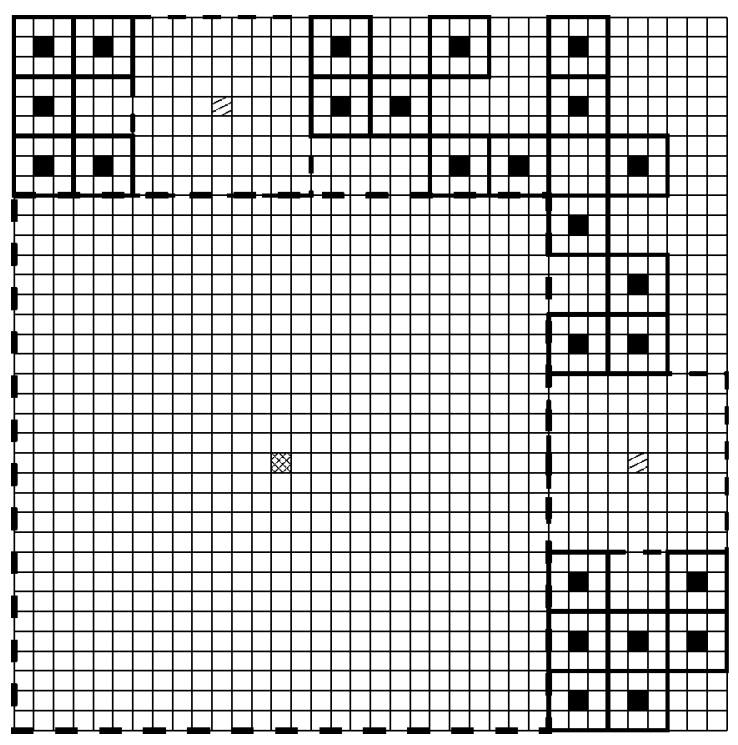

Figure 1. Artificial image with level 1,2, and 3 seeds.
- Level 1 seed pixels

Level 2 seed pixels

图 Level 3 seed pixels

where $R$ is the radiance of the hue and is defined as:

$$
R=\frac{1}{9} \sqrt{C^{2}+S^{2}} .
$$

If the value of VARHUE is lower than the threshold $T_{\mathrm{VAR}}$, then the center level $a-1$ pixel is changed to a level $a$ seed. The value of $T_{\mathrm{VAR}}$ varies depending on the level. The threshold value for each level is determined by the following formula:

$$
T_{\mathrm{VAR}}=V A R * a,
$$

where $a$ and $V A R$ are the level and an initial variance value, respectively.

\subsection{REGION GROWING}

The region growing algorithm starts with a set of seed pixels and from these grows regions by appending to each seed pixel those neighboring pixels that satisfy a homogeneity criterion. The general growing algorithm is the same for the chromatic and achromatic regions in the image. The algorithm is summarized in Figure 2. The first seed pixel is compared to its 8-connected neighbors: eight neighbors of the seed pixel. Any of the neighboring pixels that satisfy a homogeneity criterion is assigned to the first region. This neighbor comparison step is repeated for every new pixel assigned to the first region until the region is completely bounded by the edge of the image or by pixels that do not satisfy the criterion. The color of each pixel in the first region is changed to the average color of all the pixels assigned to the region. The process is repeated for the next and each of the remaining seed pixels. 


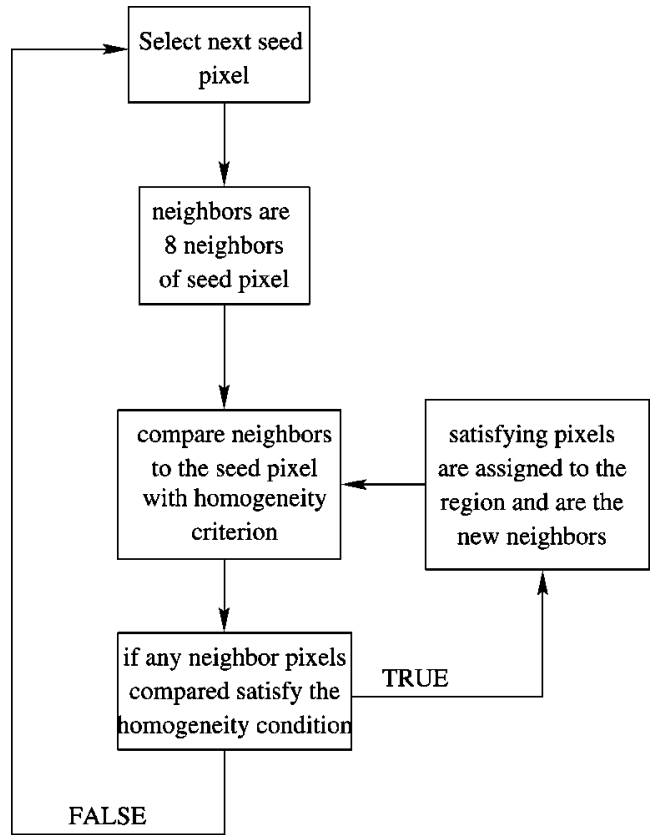

Figure 2. The region growing algorithm.

For the chromatic regions, the algorithm starts with the set of varied level seed pixels. The seed pixels in the highest level are considered first, followed by the next highest level seed pixels, and so on, until level zero seed pixels are considered. The homogeneity criterion used for comparing the seed pixel and the unassigned pixel is that, if the value of the distance metric used to compare the unassigned pixel $(i)$ and the seed pixel $(s)$ is lower than a threshold value $T_{\text {chrom}}$, then the pixel is assigned to the region. The distance measure used for comparing pixel colors is a cylindrical metric. In previous research [20], it has been found that when comparing colors the cylindrical distance metric is superior over the well known Minkowski and Canberra distance measures. The cylindrical metric computes the distance between the projections of the pixel points on a chromatic plane. It is defined as follows [42]:

$$
d_{\text {cylindrical }}(s, i)=\sqrt{\left(d_{\text {intensity }}\right)^{2}+\left(d_{\text {chromaticity }}\right)^{2}},
$$

with

$$
d_{\text {intensity }}=\left|I_{s}-I_{i}\right|
$$

and

$$
d_{\text {chromaticity }}=\sqrt{\left(S_{S}\right)^{2}+\left(S_{i}\right)^{2}-2 S_{S} S_{i} \cos \theta},
$$

where

$$
\theta= \begin{cases}\left|H_{s}-H_{i}\right| & \text { if }\left|H_{s}-H_{i}\right|<180^{\circ}, \\ 360^{\circ}-\left|H_{s}-H_{i}\right| & \text { if }\left|H_{s}-H_{i}\right|>180^{\circ} .\end{cases}
$$




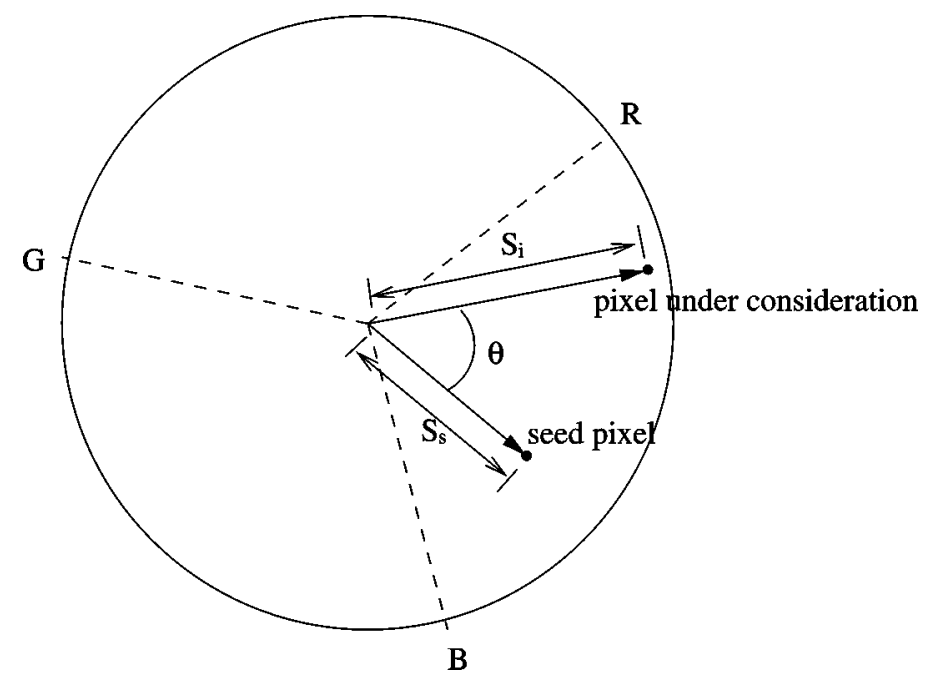

Figure 3. Chromatic plane of the HSI color space.

The value of $d_{\text {chromaticity }}$ is the distance between the 2-dimensional (hue and saturation) vectors, on the chromatic plane, of the seed pixel and the pixel under consideration, as shown in Figure 3 . Henceforth, $d_{\text {chromaticity }}$ combines both the hue and saturation (chromatic) components of the color. An examination of the metric equation (Equation (8)) shows that it can be considered as a form of the popular Euclidean distance $\left(L_{2}\right.$ norm) metric. A pixel is assigned to a region if the value of the metric $d_{\text {cylindrical }}$ is lower than a threshold $T_{\text {chrom }}$.

In the case of the achromatic pixels, the same region growing algorithm is used but with all the achromatic pixels in the image considered as level-zero seed pixels. There is no seed pixel with a level-one or higher. The seed determination algorithm is not used for the achromatic pixels because achromatic pixels constitute a small percentage in most color images. Since intensity is the only justified color atribute that can be used when comparing pixels, the homogeneity criterion used is that if the difference in the intensity values between an unassigned pixel and the seed pixel is less than a threshold value $T_{\text {achrom }}$, then the pixel is assigned to the seed pixel region. That is, if

$$
\left|I_{s}-I_{i}\right|<T_{\text {achrom }},
$$

then pixel $i$ would be assigned to the region of seed pixel $s$.

\subsection{REGION MERGING}

Regions that are similar in color but spatially disjoint will not be considered as one region in the region growing algorithm. Also, regions that are similar in color and that border each other, but are not merged because of a low growing threshold $T_{\text {chrom }}$, need to be merged. Thus, a further step is taken to merge these regions. 
A one-dimensional hue histogram is used for merging candidate regions. As mentioned previously, of the three color components of a pixel, the hue value has the greatest discriminating power because of its independence of any intensity attributes.

The algorithm determines dominant regions from the hue histogram. Dominant regions are classified as regions that have the same color as the peaks in the histogram. Once these dominant regions are determined, each remaining region is compared to them with the same color distance metric used in the region growing algorithm (Equation (8)). The merging algorithm is summarized in the following steps:

1. Determine peaks in the hue histogram of a region growing image.

2. Classify regions that have the same color as these peaks, as dominant regions.

3. Compare each of the nondominant regions with the dominant regions using the cylindrical distance metric.

4. Assign a nondominant region to the dominant region if the color distance is less than a threshold $T_{\text {merge }}$.

The color of all the pixels, in the regions assigned to a dominant region, are changed to the color of the dominant region.

\section{Experimental Results}

The performance of the proposed color image segmentation scheme was tested on a number of different images. The results on three of these images will be presented here. The original images of Claire, Carphone, and Mother_daughter are displayed in Figures 4(A), 5(A), and 6(A), respectively. These images are stills from multimedia sequences. More specifically, they are video-phone type images.

The unsupervised seed determination algorithm found seeds in the image that were in the central area of the regions in the image. It was found that increasing the variance threshold $T_{\mathrm{VAR}}$ linearly with the level (i.e., $T_{\mathrm{VAR}}=V A R * a$ ) produced the best seed pixels. Figure 4(B) shows the original Claire image with the level 3 and higher seed found pixels indicated as white pixels. Here VAR was set at 0.2. In particular, 1 level 4 and 43 level 3 seed pixels were found. It has been found that, for all the images tested, setting VAR to 0.2 gives the best results with no undesirable seeds. Figure 5(B) shows the original Carphone image with VAR set at 0.2 and the level 2 and higher seed found pixels indicated as white pixels. Here 19 level 2 seed pixels has been found. Figure 6(B) shows the original Mother_daughter image with $V A R$ set at 0.2. Here 1 level 3 (white) and 152 level 2 (black) seed pixels have been found.

Figures $4(\mathrm{C}), 5(\mathrm{C})$, and $6(\mathrm{C})$ show the three experimental images after the region growing algorithm. It was found that best results were obtained with threshold values of $T_{\text {achrom }}=15$ and $T_{\text {chrom }}=15$ which are, respectively, $15 \%$ and $7 \%$ of the maximum distance values for the achromatic and the chromatic distance measures. 

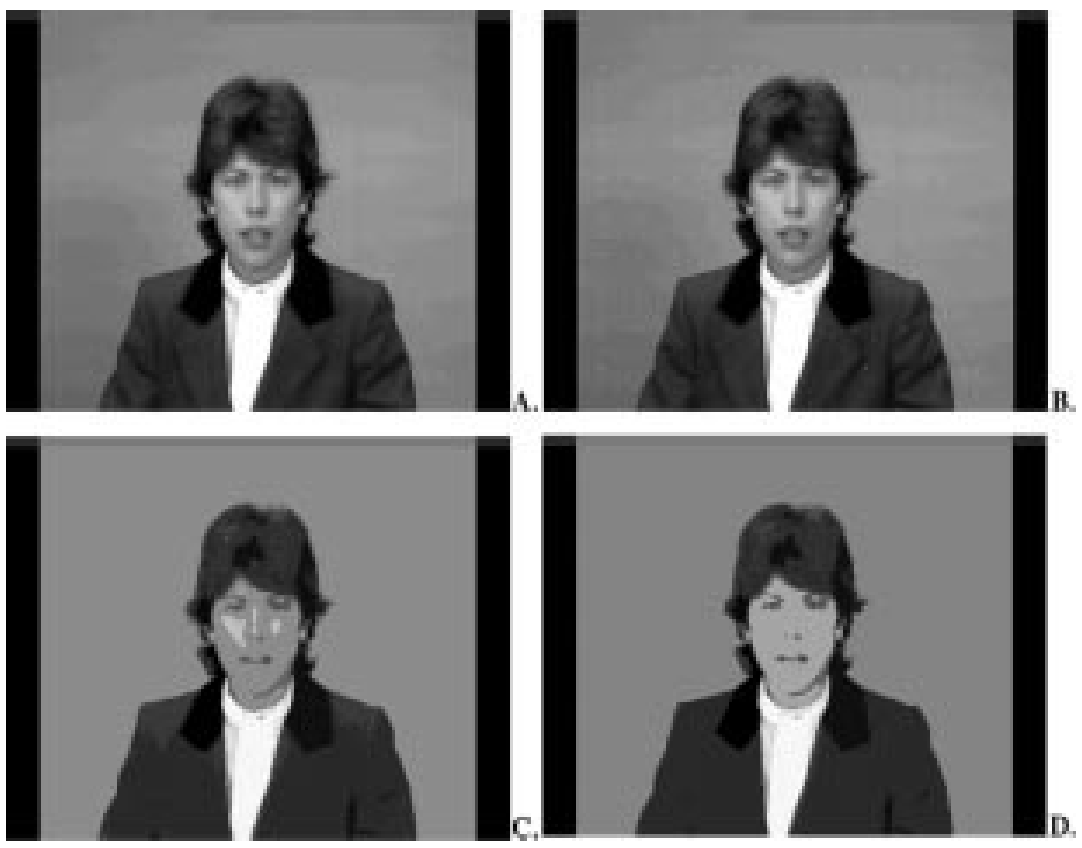

Figure 4. Claire image: original and results.
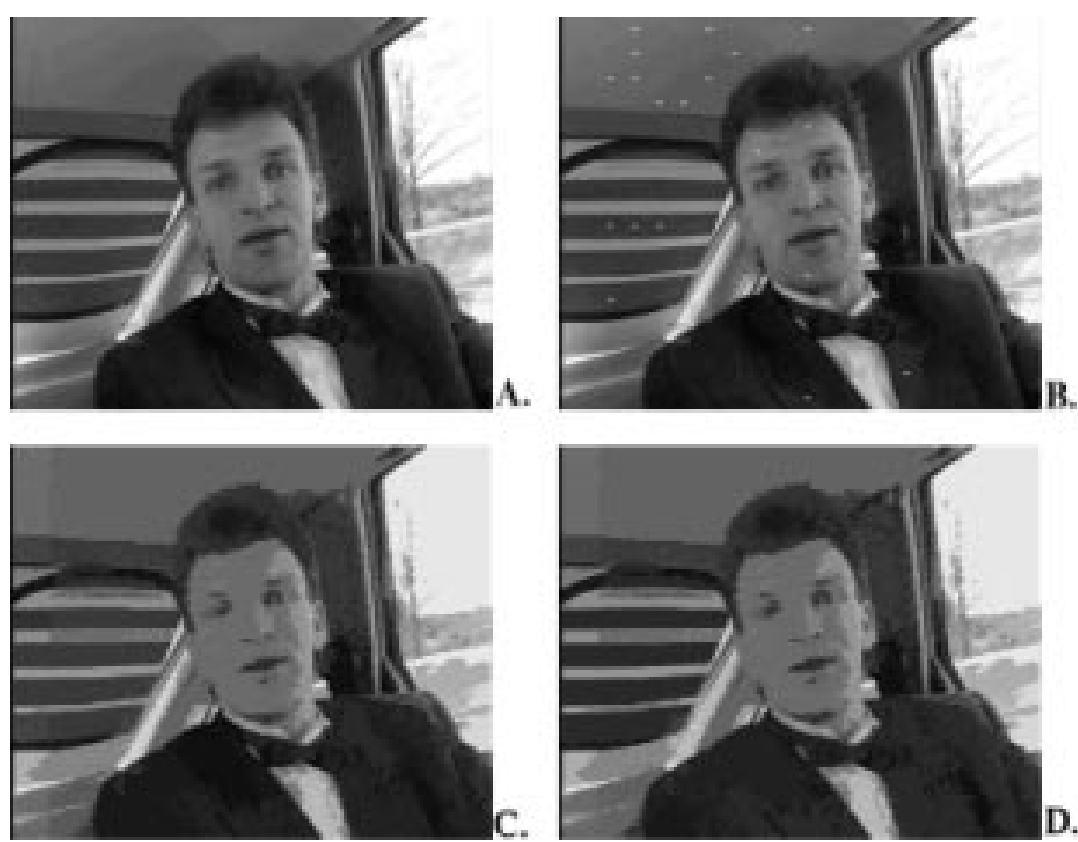

Figure 5. Carphone image: original and results. 

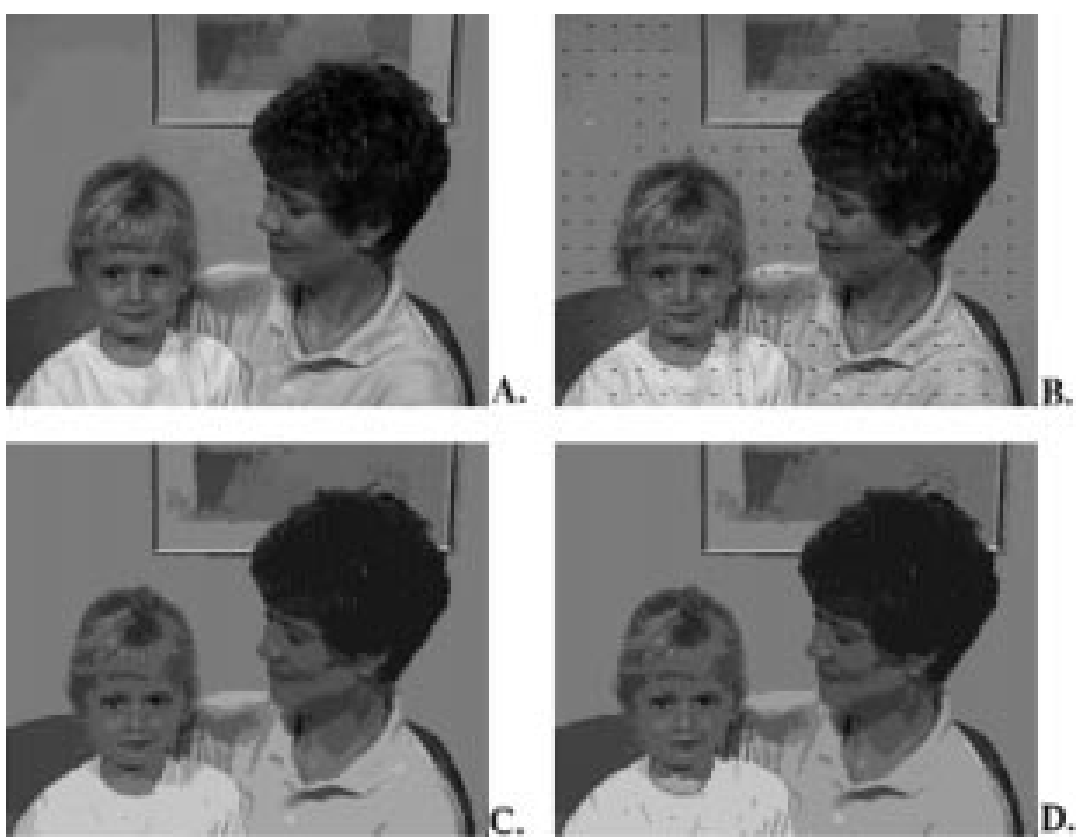

Figure 6. Mother_Daughter image: original and results.

The results show that there are regions in these segmented images that require merging.

Figures 4(D), 5(D), and 6(D) show the three experimental images after the region merging step. The threshold value $\left(T_{\text {merge }}\right)$ that gives the best merging results for a varied set of images is 20 . This is, approximately, $9 \%$ of the maximum chromatice distance value. Most of the regions that were similar in color after the region merging step are now merged.

\section{Conclusion}

Image segmentation is crucial for multimedia applications. Multimedia databases utilize segmentation for the storage and indexing of images and video. Image segmentation is used for object tracking in the new MPEG-7 video compression standard. And, as shown by the results, image segmentation is used in video conferencing for compression. These are only some of the multimedia applications in image segmentation. It is usually the first task of any image analysis process, and thus, subsequent tasks rely heavily on the quality of segmentation.

The proposed method of color image segmentation was shown to be very effective. Classifying pixels as either chromatic or achromatic avoids any color comparison of pixels that are undefined, in terms of color. The seed determination algorithm finds seed pixels that are in the center of regions which is vital when growing regions from these seeds. The cylindrical distance metric gives the best 
results when color pixels need to be compared. Merging regions that are similar in color is a final means of segmenting the image into even smaller regions.

The segmentation method proposed is interactive. We suggest the best threshold values for the segmentation scheme but these values may be easily changed for different standards. This allows for control of the degree of segmentation.

\section{References}

1. Alshatti, W. and Lambert, P.: Using eigenvecors of a vector field for deriving a second directional derivative operator for color images, in: Proc. of the 5th Internat. Conf. on Computer Analysis of Images and Patterns, Budapest, Hungary, 1993, pp. 149-156.

2. Baraldi, A., Blonda, P., Parmiggiani, F., and Satalino, G.: Contextual clustering for image segmentation, Technical Report TR-98-009, International Computer Science Institute, Berkeley, CA, 1998.

3. Celenk, M.: A color clustering technique for image segmentation, Comput. Vision Graphics Image Process. 52 (1990), 145-170.

4. Chang, M. M., Sezan, M. I., and Tekalp, A. M.: Adaptive Bayesian segmentation of colour images, J. Electronic Imaging 3(4) (1994), 404-414.

5. Chapron, M.: A new chromatic edge detector used for color image segmentation, in: Proc. of the 11th Internat. Conf. on Pattern Recognition, Vol. III, 1992, Conf. C, pp. 311-314.

6. Cohen, F. S. and Cooper, D. B.: Simple, parallel, hierarchical, and relaxation algorithms for segmenting noncausal Markovian random field models, IEEE Trans. Pattern Anal. Mach. Intelligence 9(2) (1987), 195-219.

7. Cross, G. R. and Jain, A. K.: Markov random field texture models, IEEE Trans. Pattern Anal. Mach. Intelligence 5 (1983), 25-39.

8. Cumani, A.: Edge detection in multispectral images, CVGIP: Graphical Models Image Process. 53 (1991), 40-51.

9. Derin, H. and Elliott, H.: Modeling and segmentation of noisy and textured images using Gibbs random fields, IEEE Trans. Pattern Anal. Mach. Intelligence 9(1) (1987), 39-55.

10. Di Zenzo, S.: A note on the gradient of a multi-image, Comput. Vision Graphics Image Process. 33 (1986), 116-126.

11. Fisher, N. I.: Statistical Analysis of Circular Data, Cambridge University Press, Cambridge, 1993.

12. Gauch, J. and Hsia, C.: A comparison of three color image segmentation algorithms in four color spaces, in: Proc. of the SPIE: Visual Communications and Image Processing, Vol. 1818, 1992, pp. 1168-1181.

13. Geman, S. and Geman, D.: Stochastic relaxation, Gibbs distributions, and the Bayesian restoration of images, IEEE Trans. Pattern Anal. Mach. Intelligence 6 (1984), 721-741.

14. Gong, Y. and Sakauchi, M.: Detection of regions matching specified chromatic features, Comput. Vision Image Understanding 61(2) (1995), 263-269.

15. Gonzales, R. C. and Wood, R. E.: Digital Image Processing, Addison-Wesley, Reading, MA, 1992.

16. Hartigan, J. A.: Clustering Algorithms, Wiley, New York, 1975.

17. Holla, K.: Opponent colors as a 2-dimensional feature within a model of the first stages of the human visual system, in: Proc. of the 6th Internat. Conf. on Pattern Recognition, Munich, Germany, 1982, pp. 161-163.

18. Horowitz, S. L. and Pavlidis, T.: Picture segmentation by a directed split-and-merge procedure, in: Proc. of the 2nd Internat. Joint Conf. on Pattern Recognition, Copenhagen, 1974, pp. 424 433. 
19. Huntsberger, T. L. and Descalzi, M. F.: Colour edge detection, Pattern Recogn. Lett. 3 (1985), 205-209.

20. Ikonomakis, N., Plataniotis, K. N., and Venetsanopoulos, A. N.: A region-based color image segmentation scheme, in: Proc. of the SPIE: Visual Communications and Image Processing, Vol. 3653, San Jose, CA, 1999, pp. 1202-1209.

21. Koschan, A.: A comparitive study on color edge detection, in: Proc. of the 2nd Asian Conf. on Computer Vision, ACCV'95, Vol. III, Singapore, 1995, pp. 574-578.

22. Lakshmanan, S. and Derin, H.: Simultaneous parameter estimation and segmentation of Gibbs random field using simulated annealing, IEEE Trans. Pattern Anal. Mach. Intelligence 11(8) (1989), 799-813.

23. Levine, M. D.: Vision in Man and Machine, McGraw-Hill, New York, 1985.

24. Lim, Y. W. and Lee, S. U.: On the color image segmentation algorithm based on the thresholding and the fuzzy c-means techniques, Pattern Recognition 23(9) (1990), 935-952.

25. Liu, J. and Yang, Y. H.: Multiresolution color image segmentation, IEEE Trans. Pattern Anal. Mach. Intelligence 16(7) (1994), 689-700.

26. Marr, D.: Vision, Freeman, San Francisco, CA, 1982.

27. Marr, D. and Hildreth, E.: Theory of edge detection, Proc. Roy. Soc. London (1980), 187-217.

28. Ohlander, R., Price, K., and Reddy, D. R.: Picture segmentation using a recursive splitting method, Comput. Graphics Image Process. 8 (1978), 313-333.

29. Ohta, Y., Kanade, T., and Sakai, T.: Color information for region segmentation, Comput. Graphics Image Process. 13 (1980), 222-241.

30. Pal, N. and Pal, S. K.: A review on image segmentation techniques, Pattern Recognition 26(9) (1993), 127-1294.

31. Panjwani, D. K. and Healey, G.: Markov random field models for unsupervised segmentation of textured colour images, IEEE Trans. Pattern Anal. Mach. Intelligence 17(10) (1995), 939-954.

32. Pappas, T. N.: An adaptive clustering algorithm for image segmentation, IEEE Trans. Signal Process. 40(4) (1992), 901-914.

33. Samet, H.: The quadtree and related hierarchical data structures, Computer Surveys 16(2) (1984), 187-230.

34. Scharcanski, J. and Venetsanopoulos, A. N.: Edge detection of color images using directional operators, IEEE Trans. Circuits Systems Video Technol. 7(2) (1997), 397-401.

35. Shiozaki, A.: Edge extraction using entropy operator, Comput. Vision Graphics Image Process. 33 (1986), 116-126.

36. Tominaga, S.: Color image segmentation using three perceptual attributes, in: Proc. of CVPR'86, Miami Beach, FL, USA, 1986, pp. 628-630.

37. Tominaga, S.: A color classification method for color images using a uniform color space, in: Proc. of the 10th Internat. Conf. on Pattern Recognition, Vol. 1, 1990, pp. 803-807.

38. Tou, J. and Gonzales, R. C.: Pattern Recognition Principles, Addison-Wesley, Reading, MA, 1974.

39. Trahanias, P. E. and Venetsanopoulos, A. N.: Vector order statistics operators as color edge detectors, IEEE Trans. System Man Cybernet. 26(1) (1996), 135-143.

40. Tremeau, A. and Borel, N.: A region growing and merging algorithm to color segmentation, Pattern Recognition 30(7) (1997), 1191-1203.

41. Trivedi, M. and Bezdek, J. C.: Low-level segmentation of aerial images with fuzzy clustering, IEEE Trans. Systems Man Cybernet. 16(4) (1986), 589-598.

42. Tseng, D. C. and Chang, C. M.: Color segmentation using perceptual attributes, in: Proc. of the 11th Internat. Conf. on Pattern Recognition, Vol. III, 1992, Conf. C, pp. 228-231.

43. Weeks, A. R. and Hague, G. E.: Color segmentation in the HSI color space using the K-means algorithm, in: Proc. of the SPIE, Vol. 3026, 1997, pp. 143-154. 